\title{
Mechanisms of Enhanced Phonon Scattering in Nanostructured PbTe
}

\section{Based Thermoelectric Materials}

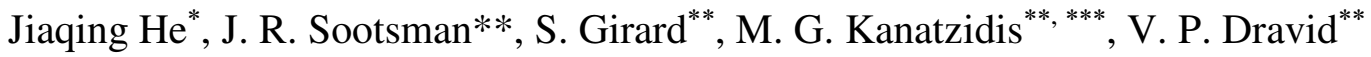 \\ *Materials Science \& Engineering, Northwestern University, Evanston Illinois 60208 \\ ${ }^{* *}$ Department of Chemistry, Northwestern University, Evanston Illinois 60208 \\ **** Materials Science Division, Argonne National Laboratory, Argonne, IL 60439
}

Thermoelectric materials hold promise in devices wherein they can convert thermal energy to electrical energy without any moving parts; they are reliable, lightweight, robust and environmentally friendly.[1] The key issue in thermoelectrics research is to develop materials with significantly increased thermoelectric figure of merit, $\mathrm{ZT}=\sigma \mathrm{S}^{2} \mathrm{~T} / \kappa$, where $\sigma$ is the electrical conductivity, $S$ is the Seebeck coefficient, and $\kappa=\kappa_{\mathrm{e}}+\kappa_{1}$ is the thermal conductivity, composed of the electronic and lattice contributions to thermal conductivity. Thus, thermoelectric performance can be improved by lowering their lattice thermal conductivity and enhancing power factor $\left(\sigma S^{2}\right)$.[2,3] In this contribution, we present S/TEM investigation of model nanostructured PbTebased thermoelectric materials coupled with calculations of the lattice thermal conductivity in order to shed light on the fundamental issue of the relationship between nanostructures and lattice thermal conductivity. Our findings have useful implications for the understanding and optimal designing of thermoelectric materials for further improvement in performance.

TEM investigation of the three samples of $\mathrm{PbTe}-2 \% \mathrm{X}(\mathrm{X}=\mathrm{Sb}$, $\mathrm{Bi}$ or $\mathrm{Pb})$ shows nanoscale precipitates with lower nanoparticle density in $\mathrm{PbTe}-\mathrm{Bi} 2 \%$ and $\mathrm{PbTe}-\mathrm{Pb} 2 \%$ samples, and with higher in $\mathrm{PbTe}-\mathrm{Sb} 2 \%$ sample. Most precipitates in all samples have a regular shape, except for $\mathrm{PbTe}-\mathrm{Pb} 2 \%$ in which we also observed some very narrow platelet-like precipitate morphology. Figure 1 shows typical low magnification images of the samples with different nanoparticle density. In Figure 1 (a) a lower density of both weak contrast spherical- and platelet-like nanoscale precipitates are observed in the $\mathrm{PbTe}-\mathrm{Pb} 2 \%$ sample. The spherical precipitates show a two-fold increase in density distribution with respect to the platelet precipitates. In Figure 1 (b), the image of the sample $\mathrm{PbTe}-\mathrm{Sb} 2 \%$ depicts higher density nanoscale precipitates with dark contrast and a size distribution of 1-10 nm. Although it is difficult to quantitatively determine the compositions of precipitates due to overlap with the matrix, we have observed higher intensity of $\mathrm{Sb}$ and $\mathrm{Bi}$ in the precipitates (dark areas) by energy dispersion x-ray spectroscopy (EDS) for samples PbTe-Sb2\% and $\mathrm{PbTe}-\mathrm{Bi} 2 \%$, respectively.

Figure 2(a) is a HRTEM image of a $4 \mathrm{~nm}$ sized precipitate in the PbTe-Sb $2 \%$ sample. One interfacial boundary $(\sim 1 \mathrm{~nm}$ dark contrast) between and matrix and precipitate is observed. Figure 2(b) is the enlarged lattice image of the boxed area, which clearly shows a dislocation at the boundary marked by oval shaped dotted line. In Figure 2(c) the IFFT image of the precipitate depicts two dislocation cores at the boundary. The Burger's circuit around the dislocation core in Figure 2(b) yields a closure failure with a projected vector $1 / 2 \mathrm{a}[010]$. A high strain region is found at the boundary region pointed by a vertical arrowhead. To analyze the strain in detail, this image was studied by geometric phase-analysis (GPA), which is a lattice image processing method for strain 
field analysis. GPA was used to investigate the variation in lattice parameter, thus the elastic strain at/around boundaries. To reduce the artifacts of the strain analysis, it is necessary to obtain and use high quality, clear lattice images. Figure $2(\mathrm{~d})$ is the strain map profile along the 001 direction $\left(\varepsilon_{\mathrm{yy}}\right)$ showing two highly strained dislocation cores, with yellow indicating expansion and blue compression (see the color scale). From the strain map distribution of this image, it is clear the arrowhead-pointed precipitate/matrix boundary is highly strained.

The presentation will highlight the subtleties of interphase interfaces and associated elastic/plastic strain distribution in reducing lattice thermal conductivity of nanostructured thermoelectrics.

\section{References:}

[1] Nolas, G. S. et al, MRS Proceedings 2004, 793,

[2] Sootsman, J. R. et al Chem. Mater. 2006, 18, 4993.

[3] This work is supported by the Office of Naval Research program.

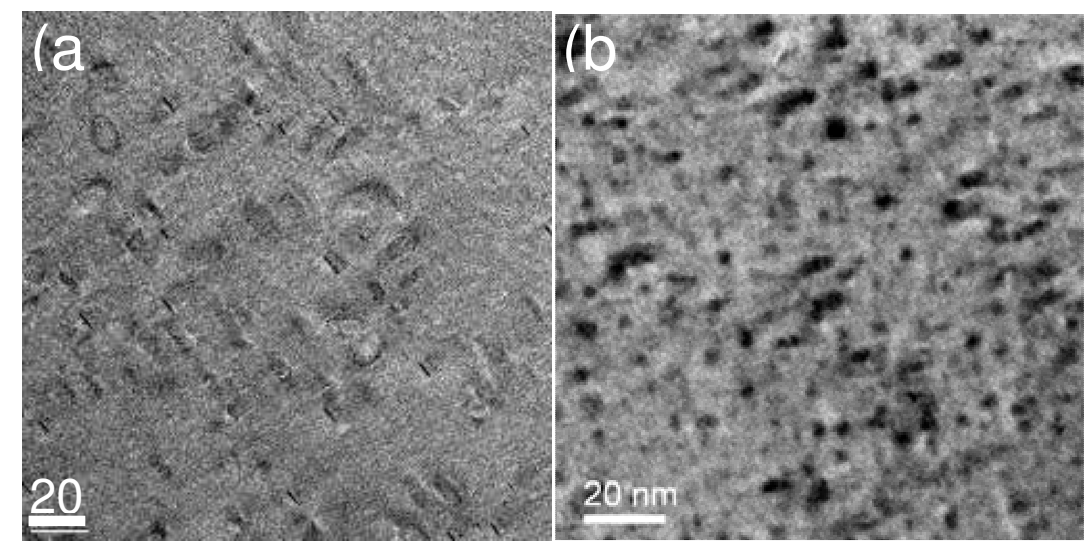

Fig.1 Typical images of two samples: PbTe-Sb $2 \%$ (a) and PbTe-Pb $2 \%$ (b). (a) The image shows two types of nanoscale precipitates: one is regular sharp; another one is platelet-like sharp. (b) The image has only one regular type of precipitate. Comparing to two images, (b) has higher nanoscale precipitate density than (a).

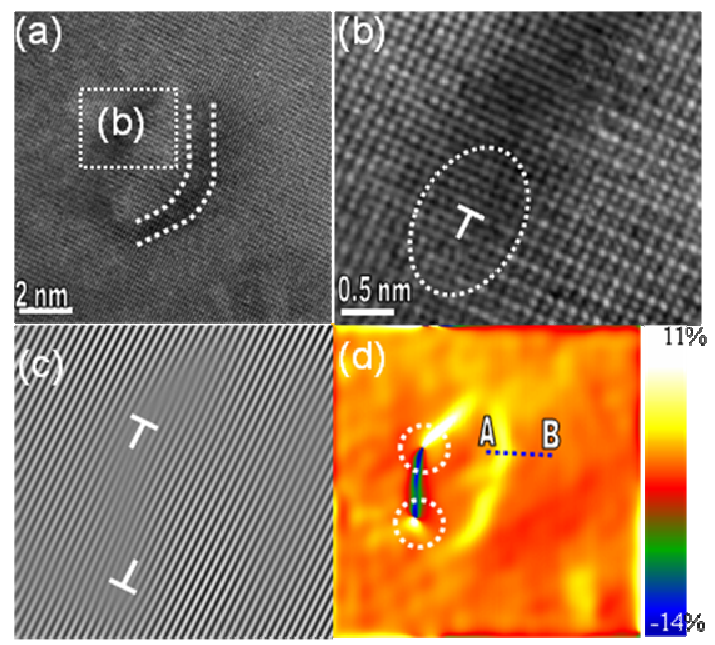

Fig.2 (a) High-magnification lattice image of $\mathrm{PbTe}-\mathrm{Sb} 2 \%$ depicts clearly boundary between precipitate and matrix, as marked in b. (b) enlarged image boxed in (a) show a misfit dislocation at the precipitate/matrix boundary. (c) IFFT image shows two dislocation cores in this precipitates. (d) The strain distribution of the nanoscale inclusions clearly show the two dislocation core in the right side and highly strained boundary in the left side. 